\title{
The impact of prostate artery embolization (PAE) on the the physical history and pathophysiology of benign prostatic hyperplasia (BPH)
}

\author{
Konstantinos Stamatiou \\ Urology Department, Tzaneio Hospital, Piraeus, Greece.
}

\begin{abstract}
Summary Aim: Prostate artery embolization (PAE) is a non invasive modality for the treatment of benign prostate hypertrophy $(\mathrm{BPH})$ related lower urinary tract symptoms (LUTS). As a relatively new procedure, data determining the clinical success is somehow scarce. In the present article we examine the current clinical outcome measures in order to identify the most accurate.

Results: Current imaging outcome measures are consistent with clinical ones only in the group of patients with adenomatous-dominant BPH while are inconsistent in patients with small sized adenomas.

Conclusions: Additional studies and/or evaluation tools are needed in order to provide accurate evaluation of clinical success in the subgroup of patients with non-adenomatous-dominant $\mathrm{BPH}$ while they may inspire new options and novel techniques for both BPH treatment and treatment-follow up.
\end{abstract}

KEY WORDS: Benign prostatic hyperplasia; Lower urinary tract symptoms; Prostate artery embolization.

Submitted 12 September; Accepted 9 December 2018

\section{INTRODUCTION}

$\mathrm{BPH}$ is a histologic diagnosis characterized by proliferation of the cellular elements of the prostate. This involves both stromal and epithelial cells, resulting in the formation of large, fairly discrete nodules in the transition zone of the prostate (1). Almost $50 \%$ of BPH patients with enlarged prostate have LUTS (2).

The last are the result of either mechanical obstruction due to glandular enlargement, or dynamic obstruction secondary to contraction of the smooth muscles of the prostate, urethra and bladder neck (3). Mild symptoms usually do not require treatment however moderate and severe symptoms could be treated with either medical therapy or surgery.

Currently prostatic arterial embolization (PAE) was emerged as a feasible procedure to treat lower urinary tract symptoms associated with BPH. PAE is the less invasive non pharmaceutical treatment. It is performed under local anaesthesia, usually by a right groin approach through the right femoral artery. It consists of selective embolization of prostatic arteries with small-diameter hydrophilic microcatheters and polyvinyl alcohol (PVA) in order to cause interruption of arterial flow. Initial studies showed that PAE led to reduction of the prostatic volume, symptom remission and improvements in quality of life However, as a relatively new procedure, data determining the accurate evaluation of clinical success of PAE is somehow scarce. In the present article we aim to investigate the potential role of elastography on the evaluation of clinical success of PAE on the treatment of benign prostatic hyperplasia (BPH).

\section{Materials AND Methods}

A search was performed in MEDLINE, NCBI, Pubmed, Cochrane Library and other electronic libraries using the terms: "prostate artery embolization AND benign prostatic hyperplasia". The articles selected were checked for the relevancy of their content to the discussed subject. The bibliographic information in the selected articles was checked for relevant publications that had not been included in the original search.

\section{RESULTS}

Since 2008 when embolization of prostatic arteries for the treatment of LUTS associated with BPH has been held for the first time, a total of 104 articles on PAE were published. After being checked for the relevancy of their content to the discussed subject, 22 papers were discarded after lecture of summary and 61 after lecture of the full paper. Finally, 21 peer-reviewed studies providing data on one or more clinical outcomes were retrieved. A recent meta-analysis of 6 large studies showed improvement in $\mathrm{Q}_{\max }$, PVR, IPSS, and QoL endpoints at 12 months, with a low incidence of serious adverse effects (0.3\%). Another recent meta-analysis of three studies comparing PAE with other treatments found greater maximum urine flow restoration and reduction in prostate volume in PAE group in relation to controls (4). Current experience shows also promising results in symptom remission and improvements in quality of life. However the overall number of PAE patients and studies meeting reliability criteria is small. Moreover, no generally accepted definition for clinical success exists (Table 1). In fact, principal outcome assessment varies among studies and could be either objective or subjective, laboratory, clinical or both. For example, regaining the ability to urinate after PAE is a measurable size whereas questionnaire-based self-reported improvement of both urination and QoL are not. Furthermore, as long as the exact mechanism by which 
Table 1.

Variability of Main outcome criteria among studies.

\begin{tabular}{|c|c|c|}
\hline Authors & N. patients & Main outcome criteria \\
\hline Li P et al, 2017 (18) & 24 & IPSS, PVR, $Q_{\max }, P S A$ \\
\hline Hwang JH et al, 2017 (19) & 9 & IPSS, PV, QoL, $Q_{\max }$ \\
\hline Little MW et al, 2017 (13) & 12 & MP-MRI, IPSS, EQ-5D-5S \\
\hline Rampoldi A et al, 2017 (20) & 43 & discontinuation of IBC, IPSS, PV, QoL, Clavien II \\
\hline Bilhim T et al, 2016 (21) & 183 & 24-hour post-PAE PSA, MRI \\
\hline Isaacson AJ et al, 2016 (22) & 12 & IPSS-QOL \\
\hline Pisco J et al, 2016 (23) & 152 & IPSS, QoL, need for additional treatment \\
\hline Wang MQ et al, 2016 (14) & 157 & IPSS, QoL, $Q_{\max }, P V, P V R$, and PSA \\
\hline Amouyal G et al, 2016 (24) & 32 & Mean IPSS, mean QoL, mean $Q_{\max }$, mean PV \\
\hline de Assis AM et al, 2015 (10) & 35 & MRI, uroflowmetry, IPSS \\
\hline Bagla S et al, 2015 (11) & 78 & AUA symptom index, QoL, or IIEF \\
\hline Russo Gl et al, 2015 (25) & 287 & IPSS, IIEF-5, PF, PVR, IPSS-QoL \\
\hline \multicolumn{3}{|c|}{$\begin{array}{l}\text { IPSS: International Prostate Symptom Score, PVR: post void residual volume, PSA: prostate-specific antigen, } \\
\text { IIEF-5: International Index of Erectile Function 5, QoL: quality of life score, TPV transitional zone prostate volume, } \\
\text { PV: prostatic volume (total volume and transition zone), EQ-5D-5S: quality of life assessment 5D-5S, } \\
\text { IBC indwelling bladder catheterization. }\end{array}$} \\
\hline
\end{tabular}

lation between PSA level elevation 24 hours after PAE and IPSS decrease at 3 months of follow-up was reported (31). It should be mentioned that other conditions that can increase PSA levels such as pre-existing inflammation, pre-treatment prostate manipulations (e.g. catheterization) and prostate size may bias this association. Moreover IPSS has inadequate sensitivity and specificity to be used as a stand-alone tool in the evaluation of clinical success of a new method such as PAE. Although, a study proposed PSA elevation after PAE to be a prognostic factor for predicting patient response to PAE (31), more research is needed in order to confirm this suggestion.

In fact, uncertainty regarding the role of pre-treatment prostatic volume in the successfulness of PAE exists. Bagla et al performed an analysis on 78 consecutive patients undergoing PAE, comparing prostate volume groups (group $1<50$

PAE affects BPH induced LUTS remains unclear, reduction in prostate volume and serum PSA value may not be the most adequate outcome measures. In fact, clinical success - in terms of IPSS and $\mathrm{Q}_{\max }$ - is not always analogous to prostate volume reduction. Moreover, the reduction on prostate volume occurs progressively and stabilized within six months of the procedure. Yet, up to $20 \%$ of patients undergoing PAE show no prostate volume reduction 3 months after the procedure (5).

A small MRI study showed that reduction of the prostate volume after embolization was significant only in patients with infarcts (6). In this study infarcts were seen in only $70.6 \%$ of the subjects, exclusively in the central gland. However, a retrospective study showed that prostatic volume decrease occurs in both central and peripheral zones (7), a fact suggesting disproportion between infarcts and reduction of the prostate volume. Although, a small MRI study proposed infarcts to be a good predictor of clinical success after PAE in patients with AUR secondary to BPH (8), it seems that it is not the case.

A significantly high PSA elevation occurs in the 24 hours after PAE. During follow-up, mean PSA decreases to a level significantly lower than at baseline. This is suspected to result from prostate inflammation and ischemia due to embolization and suggests prostate cellular apoptosis after PAE (9). However, no statistically significant correlation was detected between PSA level 24 hours after PAE and prostate volume reduction at 3 months of follow-up (10). In contrast, a statistically significant negative corre-

\section{Table 2.}

Histological and anatomical findings after PAE and their clinical significance (15).

\begin{tabular}{|ll|}
\hline Histological and anatomical findings & Clinical significance \\
\hline Fibroblast accumulation & Reparative process \\
\hline Squamous metaplasia of the surrounding epithelium & Transitional process \\
\hline Ribbons of neuthrophils, lymphocytes & Inflammatory process \\
\hline
\end{tabular}

$\mathrm{cm}^{3}$; group 2, 50-80 $\mathrm{cm}^{3}$; group $3>80 \mathrm{~cm}^{3}$ ) at baseline and follow-up to assess for differences in outcomes of American Urological Association (AUA) symptom index, quality of life (QOL)-related symptoms, and International Index of Erectile Function (IIEF). According to their result no statistically significant differences in the above parameters was found between groups (11). Other authors suggest that patients with a smaller prostate (i.e., volume < $30 \mathrm{~cm}^{3}$ ) should excluded because PAE is believed to work based on prostate volume reduction, which will be more limited in patients with almost normal sized prostates (12).

In accordance to the above, Little et al., found a statistically significant reduction in prostate volume following embolization with a median reduction of $34 \%$ (30-55) in the group of patients with adenomatous-dominant BPH (AdBPH), compared to a mean volume reduction of $22 \%$ in the non-AdBPH group. IPSS and QoL score significantly improved in the AdBPH group while there was no deterioration in sexual function in either group post-PAE (13). Similarly, Wang et al., found the clinical and imaging outcomes of PAE to be better in patients with larger prostate glands than medium-sized ones (14).

\section{Discussion}

The abovementioned findings may indicate a greater impact of PAE induced ischaemia in the adenomatous than in the stromal element of the prostate gland. However, clinical effect occurs progressively and stabilized within six months, therefore it is possible that PAE resolves dynamic obstruction also but at a slower rate. The exact mechanism by which PAE resolves dynamic obstruction is the shrinkage of the enlarged prostate gland as a result of PAE induced ischemic infarction. In contrast, the exact mechanism(s) by which PAE resolves dynamic 
obstruction remains practically unknown (15). Currently used imaging techniques are not providing relative information while knowledge on the histology of prostate tissue following PAE is extremely limited. Camara-Lopes et al., described early prostate tissue histology changes after PAE. Along with embolic material (bright eosin-red spheroids filling the vessel lumens) they observed also areas of ischemic necrosis.

The transition zone between necrotic and normal prostate tissue was characterized by inflammatory reactions containing ribbons of neuthrophils, lymphocytes and proliferated fibroblasts. Nodular fibrosis with hyalinization as a consequence of the healing process was present in some areas associated with squamous metaplasia of the epithelium lining the surrounding glands (9).

Yet there are no studies examining long term prostate tissue histology changes after PAE. However, given that PSA values decreases to a level significantly lower than at baseline but no ejaculation disorders occur it could be assumed that prostate gland return in fully functional state after PAE. As a matter of fact, metaplasia that occurs in response to necrosis and inflammation may represent an adaptive substitution of cells that are sensitive to stress by cell types better able to withstand the adverse environment and is reversible.

On the other hand, the regained ability to urinate after PAE may be associated with changes in stromal elements. Because fibroblasts are typically activated following injury and are the main producers of extracellular matrix proteins, their role as reparative cells is widely recognized (16). Fibroblasts may play a critical role in remodeling of the prostate following PAE and thus, clinical success might be also related to the regained elasticity.

The stiffness of a tissue, or its ability to resist deformation when subjected to an applied force, is indicative of the regenerative state in most organs in the body. Tissue stiffness is largely defined by chemistry and associated micro-macro structure of the extracellular matrix (ECM). Therefore, the ability to estimate ECM stiffness may assist in monitoring healing after PAE and allow estimation of clinical success.

Currently, elastic properties, of biomaterials including stiffness or shear modulus, can be investigated by elastography. The last is the only specialized imaging-based method available to spatially map strain fields, it is costeffective and safe (17). Studies comparing elastographic findings with the conventional outcome measures are needed in order to investigate the role for the elastography on the evaluation of the efficacy of PAE on the treatment of $\mathrm{BPH}$.

\section{Conclusion}

PAE is a safe and efficient method for the treatment of both mechanical and dynamic component of bladder outlet obstruction in patients with BPH. Current imaging outcome measures are consistent with clinical ones in the group of patients with adenomatous-dominant $\mathrm{BPH}$ while are inconsistent in patients with small sized adenomas. Elastography may be useful for the evaluation of PAE outcome in these patients while may shed light on the pathophysiology of $\mathrm{BPH}$ and inspire new options and novel techniques for both treatment and follow up.

\section{REFERENCES}

1. Auffenberg GB, Helfand BT, McVary KT. Established medical therapy for benign prostatic hyperplasia. Urol Clin North Am. 2009; 36:443-59.

2. Roehrborn CG. Pathology of benign prostatic hyperplasia. Int J Impot Res. 2008; 20:S11-S18.

3. Lawrentschuk N, Perera M. Benign Prostate Disorders. In: De Groot LJ, Chrousos G, Dungan K, et al., editors. Endotext [Internet]. South Dartmouth (MA): MDText.com, Inc.; 2000

4. Shim SR, Kanhai KJ, KoYM, Kim JH. Efficacy and Safety of Prostatic Arterial Embolization: Systematic Review with Meta-Analysis and Meta-Regression. J Urol. 2016: S0022-5347(16)31197-1.

5. Yoshinaga EM, Galvao O, da Motta-Leal-Filho JM, et al. Magnetic resonance analysis of prostatic volume after prostatic artery embolization (PAE) for treatment of benign prostatic hyperplasia (BPH). J Urol. 2013; 189(4S/Supplement): e820

6. Frenk NE, Baroni RH, Carnevale FC, et al. MRI findings after prostatic artery embolization for treatment of benign hyperplasia. AJR Am J Roentgenol. 2014; 203(4):813-21.

7. Lin YT, Amouyal G, Correas JM, et al.Can prostatic arterial embolisation (PAE) reduce the volume of the peripheral zone? MRI evaluation of zonal anatomy and infarction after PAE. Eur Radiol. 2016; 26:3466-73

8. Kisilevzky N, Faintuch S. MRI assessment of prostatic ischaemia: best predictor of clinical success after prostatic artery embolisation for benign prostatic hyperplasia. Clin Radiol. 2016; 71:876-82.

9. Camara-Lopes G, Mattedi R, Antunes AA, et al. The histology of prostate tissue following prostatic artery embolization for the treatment of benign prostatic hyperplasia. Int Braz J Urol. 2013; 39:222-7.

10. Assis AM, Rodrigues VCP, Yoshinaga EM, et al. Prostatic artery embolization (PAE) for treatment of benign prostatic hyperplasia in patients with prostates exceeding 90g: a prospective single center study. J Vasc Interv Radiol. 2015; 26:87-93.

11. Bagla S, Smirniotopoulos JB, Orlando JC, et al. Comparative Analysis of Prostate Volume as a Predictor of Outcome in Prostate Artery Embolization. Vasc Interv Radiol. 2015; 26:1832-8.

12. Pereira K, Halpern JA, McClure TD, et al. Role of prostate artery embolization in the management of refractory haematuria of prostatic origin. BJU Int. 2016; 118:359-65.

13. Little MW, Boardman P, Macdonald AC, et al. AdenomatousDominant Benign Prostatic Hyperplasia (AdBPH) as a Predictor for Clinical Success Following Prostate Artery Embolization: An AgeMatched Case-Control Study. Cardiovasc Intervent Radiol. 2017; 40:682-689.

14. Wang MQ, Wang Y, Yan JY, Yuan K, et al. Prostatic artery embolization for the treatment of symptomatic benign prostatic hyperplasia in men $\geq 75$ years: a prospective single-center study. World J Urol. 2016; 34:1275-83.

15. Sun F, Crisóstomo V, Báez-Díaz C, Sánchez FM. Prostatic Artery Embolization (PAE) for Symptomatic Benign Prostatic Hyperplasia (BPH): Part 2, Insights into the Technical Rationale. Cardiovasc Intervent Radiol. 2016; 39:161-9.

16. Willems IE, Havenith MG, De Mey JG, Daemen MJ. The alphasmooth muscle actin-positive cells in healing human myocardial scars. Am J Pathol. 1994; 145:868-875. 
17. Kim W, Ferguson VL. Application of Elastography for the Noninvasive Assessment of Biomechanics in Engineered Biomaterials and Tissues. Ann Biomed Eng. 2016; 44:705-724.

18. Li P, Wang C, Cao Q, Zhang J, et al. Prostatic Arterial Embolization Followed by Holmium Laser Enucleation of the Prostate as a Planned Combined Approach for Extremely Enlarged Benign Prostate Hyperplasia. Urol Int. 2017 Aug 3, [Epub ahead of print].

19. Hwang JH, Park SW, Chang IS, et al. Comparison of Nonspherical Polyvinyl Alcohol Particles and Microspheres for Prostatic Arterial Embolization in Patients with Benign Prostatic Hyperplasia. Biomed Res Int. 2017; 2017:8732351. (Epub 2017 Jun 22).

20. Rampoldi A, Barbosa F, Secco S, Migliorisi C, et al. Prostatic Artery Embolization as an Alternative to Indwelling Bladder Catheterization to Manage Benign Prostatic Hyperplasia in Poor Surgical Candidates. Cardiovasc Intervent Radiol. 2017; 40:530-536.

21. Bilhim T, Pisco J, Pereira JA, Costa NV, et al. Predictors of Clinical Outcome after Prostate Artery Embolization with Spherical and Nonspherical Polyvinyl Alcohol Particles in Patients with Benign Prostatic Hyperplasia. Radiology. 2016; 281:289-300.

22. Isaacson AJ, Raynor MC, Yu H, et al. Prostatic Artery Embolization Using Embosphere Microspheres for Prostates Measuring 80-150 cm(3): Early Results from a US Trial. J Vasc Interv Radiol. 2016; 27:709-14.

23. Pisco J, Bilhim T, Pinheiro LC, et al. Prostate Embolization as an Alternative to Open Surgery in Patients with Large Prostate and Moderate to Severe Lower Urinary Tract Symptoms. J Vasc Interv Radiol. 2016; 27:700-8.

24. Amouyal G, Thiounn N, Pellerin O, Yen-Ting L. Clinical Results After Prostatic Artery Embolization Using the PErFecTED Technique: A Single-Center Study. Cardiovasc Intervent Radiol. 2016; 39:367-75.

25. Russo GI, Kurbatov D, Sansalone S, et al. Prostatic Arterial Embolization vs Open Prostatectomy: A 1-Year Matched-pair Analysis of Functional Outcomes and Morbidities. Urology. 2015; 86:343-8.

\section{Correspondence}

Stamatiou Konstantinos, MD (Corresponding Author) stamatiouk@gmail.com

Urology Department, Tzaneio Hospital,

2 Salepoula str. 18536 Piraeus, Greece 